OPEN ACCESS

Edited by:

Albrecht Schwab,

University of Münster, Germany

Reviewed by:

Paola Italiani,

Italian National Research Council, Italy

Diana Boraschi,

Istituto di Biochimica Delle Proteine

(IBP), Italy

${ }^{*}$ Correspondence:

Wolfgang M. Kuebler

wolfgang.kuebler@charite.de

Specialty section:

This article was submitted to Molecular Innate Immunity,

a section of the journal

Frontiers in Immunology

Received: 30 November 2019

Accepted: 21 February 2020

Published: 10 March 2020

Citation:

Michalick L and Kuebler WM (2020) TRPV4-A Missing Link Between Mechanosensation and Immunity.

Front. Immunol. 11:413

doi: 10.3389/fimmu.2020.00413

\section{TRPV4-A Missing Link Between Mechanosensation and Immunity}

\author{
Laura Michalick ${ }^{1,2}$ and Wolfgang M. Kuebler ${ }^{1,2 *}$ \\ ${ }^{1}$ Institute of Physiology, Charité - Universitätsmedizin Berlin, Corporate Member of Freie Universität Berlin, \\ Humboldt-Universität zu Berlin, and Berlin Institute of Health, Berlin, Germany, ${ }^{2}$ Institute of Physiology, Berlin Institute of \\ Health, Berlin, Germany
}

Transient receptor potential vanilloid-type 4 (TRPV4) cation channel is widely expressed in all tissues as well as in immune cells and its function as mechanosensitive $\mathrm{Ca}^{2+}$ channel seems to be conserved throughout all mammalian species. Of late, emerging evidence has implicated TRPV4 in the activation and differentiation of innate immune cells, especially in neutrophils, monocytes, and macrophages. As such, TRPV4 has been shown to mediate neutrophil adhesion and chemotaxis, as well as production of reactive oxygen species in response to pro-inflammatory stimuli. In macrophages, TRPV4 mediates formation of both reactive oxygen and nitrogen species, and regulates phagocytosis, thus facilitating bacterial clearance and resolution of infection. Importantly, TRPV4 may present a missing link between mechanical forces and immune responses. This connection has been exemplary highlighted by the demonstrated role of TRPV4 in macrophage activation and subsequent induction of lung injury following mechanical overventilation. Mechanosensation via TRPV4 is also expected to activate innate immune cells and establish a pro-inflammatory loop in fibrotic diseases with increased deposition of extracellular matrix (ECM) and substrate stiffness. Likewise, TRPV4 may be activated by cell migration through the endothelium or the extracellular matrix, or even by circulating immune cells squeezing through the narrow passages of the pulmonary or systemic capillary bed, a process that has recently been linked to neutrophil priming and depriming. Here, we provide an overview over the emerging role of TRPV4 in innate immune responses and highlight two distinct modes for the activation of TRPV4 by either mechanical forces ("mechanoTRPV4") or by pathogens ("immunoTRPV4").

Keywords: TRPV4, mechanosensation, innate immunity, infection, host defense, inflammation

\section{INTRODUCTION}

Mechanotransduction is a multistep process to convert mechanical stimuli into biochemical signals that elicit specific cellular effector functions. Over the past decade many key players in this mechanosensitive machinery have been identified, e.g., ion channels like transient receptor potential vanilloid-type 4 cation channel (TRPV4) $(1,2)$, PIEZO (3-5) and epithelial $\mathrm{Na}^{+}$channel $(\mathrm{ENaC})(6)$, cellular microcompartments such as primary cilia (7) or caveolae (8), or integrins which can sense the stiffness of the extracellular matrix (ECM) (9).

In injury, infection, or cancer immune cells are attracted by biochemical cues, yet during invasion of the affected tissue they encounter changes in the biophysical properties of the microenvironment, which in turn affect their functions. This emerging role of mechanical forces in immune responses has been termed mechanoimmunology (10). Moreover, in organs with a 
high dynamic mechanical load such as the lung or the heart immune cells face rapid changes in mechanical forces during the respiratory or cardiac cycle; yet relatively little is known about the effects of such mechanical cues on the innate immune system. During cell adhesion and migration immune cells are exposed to various biophysical stimuli including shear (in the circulation as well as during cell adhesion), deformation (when squeezing though narrow capillary passages or in transmigration), or cyclic mechanical stretch (in lung and heart as a result of ventilation or cardiac function). Although mechanical forces have been shown to impact signaling during adaptive immune processes (11-13), their effects on innate immunity are rarely considered and remain poorly understood. Recent studies, however, have implicated TRPV4 function in the regulation of innate immune responses (14-16). In the present review, we connect this emerging evidence with the established role of TRPV4 in mechanosensation to propose a novel concept of mechanoimmunology via TRPV4.

\section{MechanoTRPV4}

The polymodal and non-selective TRPV4 cation channel, originally described by Strotmann et al. (17) and Liedtke et al. (18) in 2000, has been implicated over the past decades to act as a cellular mechanosensor in response to mechanical forces such as shear, stretch, osmotic swelling and shrinking, stiffening, and surface expansion (19-27) and is ubiquitously expressed in a wide range of cell types, including parenchymal cells such as smooth muscle cells, fibroblasts, epithelial cells, and endothelial cells as well as in immune cells, including macrophages, neutrophils (14, 16, 27-33).

TRPV4 activation mediates the influx of extracellular $\mathrm{Ca}^{2+}$, which can in turn activate $\mathrm{Ca}^{2+}$-triggered signaling cascades resulting in changes in transcription, vesicular transport, or cytoskeletal remodeling. The molecular mechanism how TRPV4 is activated by mechanical forces is currently not completely understood. At present, models for either direct or indirect mechanical activation of TRPV4 have been proposed $(34,35)$. The concept of direct activation is based on the assumption that an initial deformation of the plasma membrane's lipid bilayer will cause an expansion in cross sectional area, which creates a membrane tension-dependent energy difference followed by conformational change of the ion channel and thereby promotes force activation, as previously described for KCNK4 potassium channels by Brohawn et al. (36). This concept is supported by studies of Loukin et al. (37) who showed that TRPV4 can be activated by pipette suction in the presence of enzyme inhibitors in Xenopus oocytes, thus excluding mechanisms of indirect activation (37).

The concept of indirect TRPV4 activation follows the notion that TRPV4 is rather mechanically gated via intracellular signaling pathways such as integrin signaling, intracellular messengers and kinases, or simply by changes in surface expression $(38,39)$. Therefore, it has been demonstrated that forces applied to $\beta_{1}$-integrins result in ultra-rapid activation of $\mathrm{Ca}^{2+}$ influx through TRPV4 channels and that the TRPV4 channels are rather activated by mechanical strain in the cytoskeletal backbone of the focal adhesion than by deformation of the lipid bilayer or peripheral cortical cytoskeleton (40). Such localized indirect activation is proposed to cause highly compartmentalized TRPV4-mediated $\mathrm{Ca}^{2+}$ signaling at focal adhesions and facilitates downstream activation of additional $\beta_{1}$-integrins (integrin-to-integrin signaling) and leads to cell reorientation $(40,41)$. Moreover, several studies have shown that TRPV4 activation in response to osmotic or mechanical stress depends on formation of intracellular mechanomessengers, like lipid metabolites as arachidonic acid and its derivative $5^{\prime}, 6^{\prime}$ epoxyeicosatrienoic acid, and $\mathrm{PIP}_{2}$ (21, 42-45). Additionally, calmodulin as a classical second messenger binds to TRPV4 and mediates $\mathrm{Ca}^{2+}$ influx by conformational change and dissociation of its $\mathrm{N}$ - and C-terminus (20). It also has been observed that several protein kinases affect the activity of TRPV4 and/or facilitates binding to anchoring proteins (AKAPs) and F-actin and stabilize the channel in the plasma membrane $(24,27,46-$ 48). As such, a series of intracellular signaling cascades have been identified that modulate TRPV4 activity and may serve as pathways for indirect TRPV4 activation by mechanical forces.

In addition to the intracellular signaling pathways activating TRPV4, mechanical forces can affect TRPV4 trafficking and upregulate surface expression of the channel by recruitment from intracellular pools of TRPV4 to the plasma membrane via mechanoreceptive structures like calveolae, integrins, or adherens junctions $(8,26,32,41)$. Subcellular localization and trafficking of TRPV4 have been proposed to depend on preand post-translational modifications, like alternative splicing (49), nitrosylation, glycosylation and phosphorylation (27, 47, 50, 51). As these mechanisms were, however, largely identified using fluorescent-tagged overexpression systems their exact role in the trafficking of endogenous TRPV4 still remains incompletely understood.

In summary, TRPV4 may not only respond to diverse triggers via various modes (direct and indirect) of activation, diverse signaling pathways and protein modifications, but also different mechanisms to increase the abundance of open $\mathrm{Ca}^{2+}$ channels at the plasma membrane, respectively (Figure 1).

\section{ImmunoTRPV4}

The role of TRPV4 in the innate immune system was first recognized more than a decade ago due to its thermosensitivity. Increments in body temperature in response to infection (i.e., fever) are important activators of the immune system with an evolutionary conserved role in host defense $(52,53)$. TRPV4 became first implicated in thermo-dependent immune modulation based on its role in thermal hyperalgesia (5456). At the same time, similar effects were reported for other $\mathrm{Ca}^{2+}$ channels, such as the temperature-dependent activation of stromal interaction molecule (STIM) 1, which induces $\mathrm{Ca}^{2+}$ influx and in turn modulates gene expression and immune functions (57). These studies unveiled for the first time a direct link between $\mathrm{Ca}^{2+}$ channels, temperature, and immune function. 


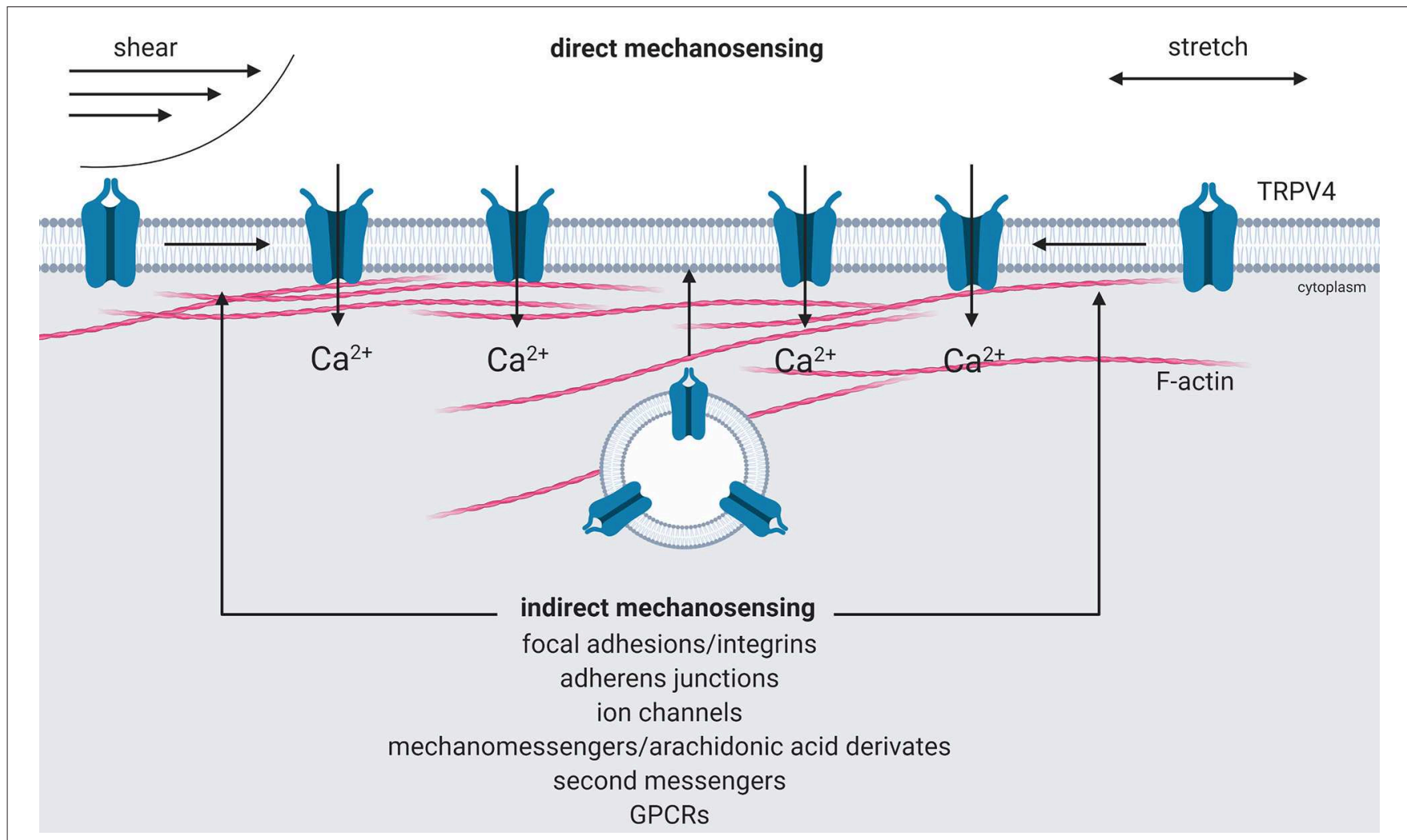

FIGURE 1 | Activation of mechanoTRPV4. Direct activation of TRPV4 by shear or stretch forces results in an expansion in cross sectional area that creates a tension-dependent energy difference and leads to conformational changes of the channel by force activation (direct mechanosensing). Indirect activation is mediated by intracellular signaling cascades triggered via mechanosensitive focal adhesions or adherens junctions, ion channels, by intracellular mechano- or second messengers, G-protein-coupled receptors, e.g., protease-activated receptors that either activate TRPV4 or recruit it from intracellular pools to the plasma membrane (indirect mechanosensing). Created with BioRender.com.

In 2008, Spinsanti and colleagues for the first time detected high expression levels of TRPV4 in human leukocytes (58). Subsequent functional studies from our group identified an important role for TRPV4 in regulating key neutrophil functions in response to pro-inflammatory stimuli like production of reactive oxygen species, cell adhesion, or migration (16). In vivo, Trpv4-deficient mice showed a marked protection from acute lung injury in two independent studies following either acidinduced or chlorine-induced lung injury $(16,59,60)$. These effects were replicated by pharmacological inhibition of TRPV4, which similar to Trpv4 deficiency attenuated characteristic signs of lung injury including hypoxemia, reduced compliance, edema formation, histological evidence of lung injury, and last not least, neutrophil infiltration and the release of proinflammatory cytokines $(27,59)$. Bone marrow chimeras from Trpv4-deficient and corresponding wild type mice revealed that the barrier protective effects in Trpv4-deficient mice was mostly attributable to a lack of TRPV4 in parenchymal tissue (presumably most relevant in endothelial cells), whereas TRPV4 deficiency in hematopoietic blood cells primarily reduced neutrophil infiltration into the injured lung (16). As such, it remains to be shown to which extent TRPV4-mediated activation of neutrophils affects organ function in vivo. In principle, these findings demonstrate that TRPV4 regulates neutrophil adhesion and migration, whereas in barrier forming cells like endothelial and epithelial cells, TRPV4 acts as a door opener for protein and fluid extravasation.

In macrophages, TRPV4 mediates both pro-inflammatory functions including phagocytosis, adhesion, and reactive oxygen species production, as well as anti-inflammatory effects and secretion of pro-resolution cytokines and bacterial clearance $(14,15)$. As such, macrophage TRPV4 may exert both protective and detrimental effects to the host tissue, by facilitating bacterial clearance in infection while promoting parenchymal injury in sterile inflammation $(16,27,59)$. In a recent study, a similar double-edged role of mechanosennsation in the modulation of the innate immune response to sterile inflammation vs. bacterial infection was reported for another emerging mechanoimmunological cation channel, PIEZO1 (5).

An important additional role of TRPV4 in innate immunity relates to expression and function in the vascular endothelium, which by way of lining the inner surface of blood vessels and regulating cell adhesion and migration via expression of adhesion molecules acts as a gate keeper and controls the access for cells of the innate (and adaptive) immune system to sites of inflammation (61). TRPV4 activation in lung endothelial cells has 
been shown to increase vascular permeability $(16,27,29)$, in part via disintegration of cell junctions (62) and degradation of ECM components and non-matrix components like integrins and VEcadherins by matrix metalloproteinases (MMPs) MMP2 and MMP9 (63), and in part by calmodulin-dependent activation of the endothelial contractile machinery (64). As a consequence of these effects, TRPV4 activation results in endothelial detachment from the basal lamina, consecutive disruption of the endothelial barrier, and ultimately edema formation $(65,66)$. These effects seem to be most prominent in the pulmonary microvasculature (67), which may be related to TRPV4's roles in immunity and defense on the one, and the fact that the alveolo-capillary barrier presents a large surface for pathogen invasion on the other hand.

In lung alveolar epithelium, TRPV4 has been shown to act as a critical regular of epithelial barrier function, but at the same time revealed protective effects by increasing in bacterial clearance in large airways $(31,68,69)$. All these findings identify TRPV4 as an important regulator of innate host defense responses including the regulation of phagocytes such as neutrophils and macrophages, but also of barrier forming epithelial and endothelial cells.

\section{TRPV4 IN HOST DEFENSE}

In line with its role in innate immune cells, TRPV4 has been implicated in different scenarios of host defense. In a murine model of Streptococcus pneumoniae infection TRPV4-deficiency prevented leukocyte infiltration, reduced bacterial load in the alveolar space, and attenuated characteristic features of lung injury (70). While these data unequivocally highlight the role of TRPV4 in host defense against gram-positive bacteria, the mechanism of TRPV4 activation by such bacteria or their pore-forming toxins such as pneumolysin (S. pneumoniae), $\alpha$ hemolysin (S. aureus), or listeriolysin O (L. monocytogenes) is still unclear (70-72).

In gram-negative infections with bacteria such as Escherichia coli and Pseudomonas aeruginosa, TRPV4 activation by ECM stiffening during infection synergizes with LPS-stimulated TLR4 activation of p38 and thereby promotes host defense and resolution from lung injury $(15,73)$. Conversely, activation of protease-activated receptor (PAR)-2 by thrombin suppresses TRPV4 activity in macrophages and resolves lung injury (74). Similarly, PARs are also activated by neutrophil elastase (NE), matrix metalloproteases (MMPs) or other microbial proteases (33, 75-79) which has been implicated to degrade ECM and thereby causing remodeling and matrix stiffening during infection $(33,63,73,80)$.

Albeit the number of studies on TRPV4 in immune cells is still limited, TRPV4 emerges as a regulator of innate immunity and host defense and may sense mechanical changes of the extracellular environment during inflammation. Therefore, occurring mechanical forces are crucial for TRPV4-mediated immune response and regulate both pro- and anti-inflammatory effects, which may have both beneficial effects in terms of bacterial clearance and resolution from injury.

\section{TRPV4 IN MECHANOSENSATION OF IMMUNE CELLS}

Mechanical forces generated by hemodynamic forces or a factor of ECM composition under physiological and pathophysiological conditions are acting on immune cells and can be subclassified in (i) mechanical stretch by shape changes during cell passage through narrow capillary segments, (ii) shear stress acting on circulating or adherent immune cells as a function of blood flow or viscosity, and (iii) changes in substrate stiffness of the ECM induced by inflammation $(81,82)$.

In particular, mechanical stretch has been implicated as a central component in pathological processes at the alveolocapillary barrier of the lung where it has been extensively studied in the context of ventilator-induced lung injury (VILI). In endothelial and epithelial cells, TRPV4 has been shown to become activated during mechanical stretch as exerted by mechanical (over-) ventilation leading to $\mathrm{Ca}^{2+}$ influx and subsequent loss of lung barrier integrity and the release of cytokines $(27,29,68)$. For macrophages, TRPV4 function has been shown to be critical in the pro-inflammatory response to mechanical ventilation (14). As shown by Hamanaka and colleagues in studies on isolated-perfused mouse lungs, replacement of wild type with Trpv4-deficient macrophages in wild type lungs was sufficient to attenuate classical features of VILI, a finding that was linked to stretch-induced and TRPV4dependent intracellular $\mathrm{Ca}^{2+}$ signaling, and the subsequent formation of reactive oxygen and nitrogen species in vitro.

In neutrophils, transmigration during VILI has so far largely been considered as a response secondary to the mechanical stretch on parenchymal cells $(16,83)$. In contrast to the systemic circulation where neutrophil adhesion and migration are primarily localized to postcapillary venules and mediated by adhesion molecules (61), the initial mechanism of neutrophil sequestration in the lung is largely based on cytoskeletal rearrangement and formation of F-actin rims which increase cellular stiffness and as a result, decrease their ability to change their shape from spherical to elliptical. These changes in deformability prevents activated neutrophils to pass through the narrow capillary segments of the alveolo-capillary network where they get trapped at sites of inflammation (84). While neutrophil stiffening was originally considered an irreversible feature, recent studies suggest that alternating neutrophil stiffening and softening can drive the dynamic oscillation of neutrophils between the activated/primed and deactivated/deprimed state (85). Since F-actin has been shown to bind to activated TRPV4, it can be speculated that this priming/depriming occurs as a function of TRPV4 activation secondary to the formation of F-actin rims (24). Given the implications of such mechanical effects due to e.g., changes in neutrophil shape and stiffness not only on neutrophil kinetics through the vascular system but also on their biological responsiveness in health and disease (86), the molecular dissection of the underlying signaling pathways and the potential link to TRPV4 mechanosensation may be of considerable scientific interest and relevance. 


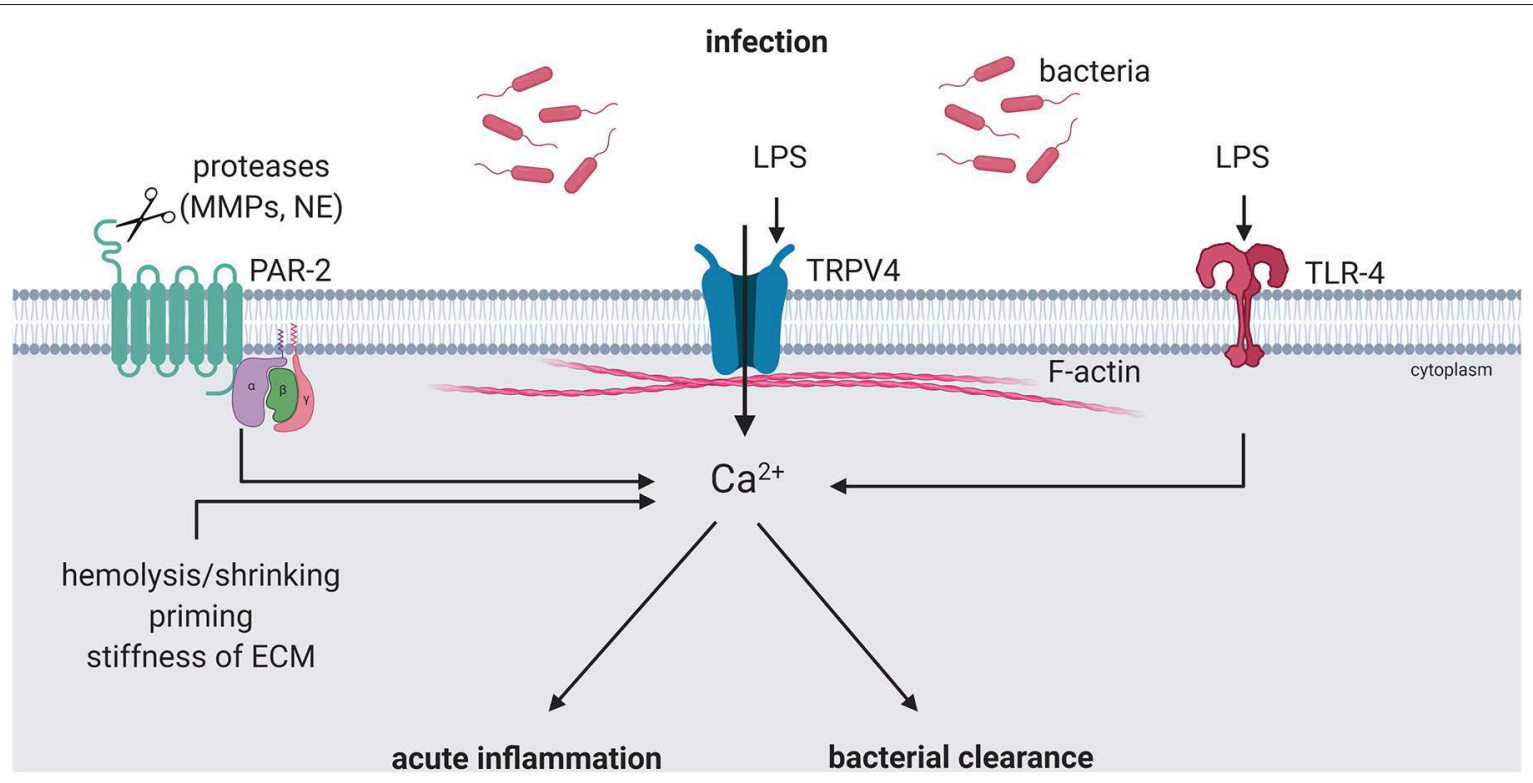

FIGURE 2 | ImmunoTRPV4 activation during infection. Pathogen-associated signals, e.g., lipopolysaccharide (LPS) can modulate TRPV4 function directly or indirectly. Direct activation can lead to pro-inflammatory phenotypes and boost the response in acute inflammation. Activation of TLR-4 by LPS and cleavage of PAR-2 by release matrix metalloproteases (MMPs) and/or neutrophil elastase (NE) during inflammation can modulate the TRPV4-mediated immune response toward an anti-inflammatory phenotype and induce bacterial clearance. Created with BioRender.com.

Shear stress in the vasculature is a result of blood flow velocity, vessel diameter, and blood viscosity and primarily acts on endothelial cells outlining the vessel lumen. Endothelial cells respond to shear stress by segregation of TRPV4 channels from $\beta$-catenin following relocating TRPV4 from adherens junctions to focal adhesions of the basal membrane which in turn increases endothelial permeability by destabilization of junctional complexes and $\mathrm{Ca}^{2+}$-mediated cytoskeletal remodeling (87). While it is reasonable to expect that circulating immune cells tethering or adhering to the vascular wall experience likewise considerable degrees of vascular shear stress, the effects of fluid shear stress on innate immune cells have so far not been extensively addressed. In the alveolar compartment, alveolar macrophages have been shown to contribute to VILI by secretion of pro-inflammatory mediators in a TRPV4-dependent manner (14). This effect is presumably predominantly caused by stretch rather than shear effects. Nevertheless, it is conceivable that shear-dependent activation of macrophages may become relevant in conditions of alveolar fluid accumulation when fluid will cyclically shift in and out of the alveolus resulting in considerable shear forces exerted not only on alveolar epithelial cells but also on alveolar macrophages $(88,89)$.

Finally, substrate stiffness is regulated by the composition of the ECM which changes as a function of physiological (development, aging) and pathophysiological (atherosclerosis, hypertension, fibrosis) processes (90-93). TRPV4 has been identified as a major mechanosensor for substrate stiffness, but so far this function has been exclusively attributed to parenchymal cells $(30,31,94-96)$. Yet, it is fair to speculate that changes in substrate stiffness will similarly affect the mechanical forces that act upon immune cells during the processes of adhesion and transmigration, which accordingly may affect TRPV4-dependent cellular responses. Conversely, TRPV4-mediated activation of immune cells may in turn affect local ECM structure and composition by secretion of MMPs. As such, TRPV4 may play an important role in proteolytic disruption of ECM, cell-cell, and cell-matrix interaction by MMPs that is required for effective immune cell extravasation to sites of injury and inflammation (63). By similar mechanisms, TRPV4 may also contribute to chronic parenchymal remodeling, explaining its prominent role in tissue fibrosis in a positive feedback of substrate stiffening and TRPV4-mediated pro-fibrotic effects $(30,97)$.

In line with a critical role of substrate stiffness for immune cell function, the $\mathrm{Ca}^{2+}$ response to LPS in of macrophages were shown to correlate with substrate stiffness. Notably, such substrate stiffness-dependent modulation of macrophage signaling can alter macrophage phenotype toward an antiinflammatory phenotype (M2) initiating bacteria clearance and resolution of lung injury $(15,73)$.

\section{SUMMARY AND CONCLUSION}

This review provides an update on the role of TRPV4 in mechanosensation ("mechanoTRPV4") on the one and inflammation and host defense ("immunoTRPV4") on the other hand with the aim to point toward a possible, albeit still speculative, role of TRPV4 in mechanoimmunology.

Activation of mechanoTRPV4 directly impacts host defense in that it reduces endothelial and epithelial barrier function, but 
at the same time promotes the infiltration of innate immune cells and the release of pro- and anti-inflammatory cytokines $(15,16,27)$. In addition, TRPV4 activation can regulate the delivery of circulating immune cells to local sites of inflammation and infection by mediating vasodilation $(98,99)$. Activation of immunoTRPV4 triggers and promotes inflammation and has emerged as a key regulator of bacterial clearance. Importantly, mechanosensitive and immunoregulatory functions of TRPV4 may not be distinct, but intrinsically linked, thus opening a new view on mechanoregulation of immune responses. This concept has already been well-established for epithelial and endothelial cells, where mechanical activation of TRPV4 has emerged a major regulator of barrier function and inflammatory responses. Yet, this notion has also been demonstrated for innate immune cells such as macrophages where $\mathrm{Ca}^{2+}$ signaling as a function of TRPV4-mediated sensing of substrate stiffness has been shown to shift the immune response from pro-inflammatory to an anti-inflammatory and resolving phenotype $(15,73)$ (Figure 2). Similar scenarios of mechanoregulation of immune cell function via TRPV4 may relate to a variety of scenarios where immune cells undergo changes in mechanical stress, such as during cell

\section{REFERENCES}

1. Liedtke W, Tobin DM, Bargmann CI, Friedman JM. Mammalian TRPV4 (VR-OAC) directs behavioral responses to osmotic and mechanical stimuli in Caenorhabditis elegans. Proc Natl Acad Sci USA. (2003) 100(Suppl 2):145316. doi: 10.1073/pnas.2235619100

2. Liedtke WB. TRPV channels' function in osmo- and mechanotransduction. In: TRP Ion Channel Function in Sensory Transduction and Cellular Signaling Cascades, eds W. B. Liedtke, S. Heller (Boca Raton, FL: CRC Press/Taylor \& Francis). Available online at: http://www.ncbi.nlm.nih.gov/books/NBK5262/ (accessed September 21, 2016).

3. Zhao Q, Wu K, Geng J, Chi S, Wang Y, Zhi P, et al. Ion permeation and mechanotransduction mechanisms of mechanosensitive piezo channels. Neuron. (2016) 89:1248-63. doi: 10.1016/j.neuron.2016.01.046

4. Ihara T, Mitsui T, Nakamura Y, Kanda M, Tsuchiya S, Kira S, et al. The oscillation of intracellular $\mathrm{Ca}^{2+}$ influx associated with the circadian expression of Piezol and TRPV4 in the bladder urothelium. Sci Rep. (2018) 8:5699. doi: 10.1038/s41598-018-23115-w

5. Solis AG, Bielecki P, Steach HR, Sharma L, Harman CCD, Yun S, et al. Mechanosensation of cyclical force by PIEZO1 is essential for innate immunity. Nature. (2019) 573:69-74. doi: 10.1038/s41586-019-1485-8

6. Guo D, Liang S, Wang S, Tang C, Yao B, Wan W, et al. Role of epithelial Na+ channels in endothelial function. J Cell Sci. (2016) 129:2907. doi: $10.1242 /$ jcs. 168831

7. Spasic M, Jacobs CR. Primary cilia: cell and molecular mechanosensors directing whole tissue function. Semin Cell Dev Biol. (2017) 71:4252. doi: 10.1016/j.semcdb.2017.08.036

8. Saliez J, Bouzin C, Rath G, Ghisdal P, Desjardins F, Rezzani R, et al. Role of caveolar compartmentation in endothelium-derived hyperpolarizing factor-mediated relaxation: $\mathrm{Ca}^{2+}$ signals and gap junction function are regulated by caveolin in endothelial cells. Circulation. (2008) 117:106574. doi: 10.1161/CIRCULATIONAHA.107.731679

9. Ringer P, Colo G, Fässler R, Grashoff C. Sensing the mechanochemical properties of the extracellular matrix. Matrix Biol. (2017) 64:6-16. doi: 10.1016/j.matbio.2017.03.004

10. Pageon SV, Govendir MA, Kempe D, Biro M. Mechanoimmunology: molecular-scale forces govern immune cell functions. Mol Biol Cell. (2018) 29:1919-26. doi: 10.1091/mbc.E18-02-0120 adhesion (shear stress) and transmigration (substrate stiffness, shape change), capillary transit (shape change) as well as tissue strain e.g., in mechanically ventilated lungs (stretch). So far, the link between mechanoTRPV4 and immunoTRPV4 has not been characterized in detail, but may provide for important insights into the regulation of innate immunity and host defense and as such, for the development of novel preventive, therapeutic, or adjuvant strategies in inflammatory and infectious diseases such as sepsis, pneumonia, or sterile inflammation as in VILI.

\section{AUTHOR CONTRIBUTIONS}

All authors listed have made a substantial, direct and intellectual contribution to the work, and approved it for publication.

\section{ACKNOWLEDGMENTS}

We acknowledge support from the German Research Foundation (DFG) and the Open Access Publication Fund of Charité Universitätsmedizin Berlin.
11. Kim ST, Takeuchi K, Sun Z-YJ, Touma M, Castro CE, Fahmy A, et al. The alphabeta T cell receptor is an anisotropic mechanosensor. J Biol Chem. (2009) 284:31028-37. doi: 10.1074/jbc.M109.052712

12. Reinherz EL. The structure of a T-cell mechanosensor. Nature. (2019) 573:502-4. doi: 10.1038/d41586-019-02646-w

13. Harrison DL, Fang Y, Huang J. T-cell mechanobiology: force sensation, potentiation, and translation. Front Phys. (2019) 7:45. doi: 10.3389/fphy.2019.00045

14. Hamanaka K, Jian M-Y, Townsley MI, King JA, Liedtke W, Weber DS, et al. TRPV4 channels augment macrophage activation and ventilatorinduced lung injury. Am J Physiol Lung Cell Mol Physiol. (2010) 299:L35362. doi: 10.1152/ajplung.00315.2009

15. Scheraga RG, Abraham S, Niese KA, Southern BD, Grove LM, Hite RD, et al. TRPV4 Mechanosensitive ion channel regulates lipopolysaccharide-stimulated macrophage phagocytosis. J Immunol. (2016) 196:428-36. doi: 10.4049/jimmunol.1501688

16. Yin J, Michalick L, Tang C, Tabuchi A, Goldenberg N, Dan Q, et al. Role of transient receptor potential Vanilloid 4 in neutrophil activation and acute lung injury. Am J Respir Cell Mol Biol. (2016) 54:37083. doi: 10.1165/rcmb.2014-0225OC

17. Strotmann R, Harteneck C, Nunnenmacher K, Schultz G, Plant TD. OTRPC4, a nonselective cation channel that confers sensitivity to extracellular osmolarity. Nat Cell Biol. (2000) 2:695-702. doi: 10.1038/35036318

18. Liedtke W, Choe Y, Martí-Renom MA, Bell AM, Denis CS, Sali A, et al. Vanilloid receptor-related osmotically activated channel (VR-OAC), a candidate vertebrate osmoreceptor. Cell. (2000) 103:525-35. doi: 10.1016/S0092-8674(00)00143-4

19. Liedtke W, Friedman JM. Abnormal osmotic regulation in trpv4-/- mice. Proc Natl Acad Sci USA. (2003) 100:13698-703. doi: 10.1073/pnas.1735416100

20. Strotmann R, Schultz G, Plant TD. $\mathrm{Ca}^{2+}$-dependent potentiation of the nonselective cation channel TRPV4 is mediated by a C-terminal calmodulin binding site. J Biol Chem. (2003) 278:26541-9. doi: 10.1074/jbc.M302590200

21. Vriens J, Watanabe H, Janssens A, Droogmans G, Voets T, Nilius B. Cell swelling, heat, and chemical agonists use distinct pathways for the activation of the cation channel TRPV4. Proc Natl Acad Sci USA. (2004) 101:396401. doi: 10.1073/pnas.0303329101

22. O'Neil RG, Heller S. The mechanosensitive nature of TRPV channels. Pflugers Arch. (2005) 451:193-203. doi: 10.1007/s00424-005-1424-4 
23. Wu L, Gao X, Brown RC, Heller S, O'Neil RG. Dual role of the TRPV4 channel as a sensor of flow and osmolality in renal epithelial cells. Am J Physiol Renal Physiol. (2007) 293:F1699-713. doi: 10.1152/ajprenal.00462.2006

24. Shin SH, Lee EJ, Hyun S, Chun J, Kim Y, Kang SS. Phosphorylation on the Ser 824 residue of TRPV4 prefers to bind with F-actin than with microtubules to expand the cell surface area. Cell Signal. (2012) 24:64151. doi: 10.1016/j.cellsig.2011.11.002

25. Jo AO, Ryskamp DA, Phuong TTT, Verkman AS, Yarishkin O, MacAulay $\mathrm{N}$, et al. TRPV4 and AQP4 channels synergistically regulate cell volume and calcium homeostasis in retinal müller glia. J Neurosci. (2015) 35:1352537. doi: 10.1523/JNEUROSCI.1987-15.2015

26. Baratchi S, Almazi JG, Darby W, Tovar-Lopez FJ, Mitchell A, McIntyre P. Shear stress mediates exocytosis of functional TRPV4 channels in endothelial cells. Cell Mol Life Sci. (2016) 73:649-66. doi: 10.1007/s00018-015-2018-8

27. Michalick L, Erfinanda L, Weichelt U, van der Giet M, Liedtke W, Kuebler WM. Transient receptor potential Vanilloid 4 and serum glucocorticoid-regulated kinase 1 are critical mediators of lung injury in overventilated mice in vivo. Anesthesiology. (2017) 126:300-11. doi: 10.1097/ALN.0000000000001443

28. Jia Y, Wang X, Varty L, Rizzo CA, Yang R, Correll CC, et al. Functional TRPV4 channels are expressed in human airway smooth muscle cells. Am J Physiol Lung Cell Mol Physiol. (2004) 287:L272-8. doi: 10.1152/ajplung.00393.2003

29. Hamanaka K, Jian M-Y, Weber DS, Alvarez DF, Townsley MI, Al-Mehdi $\mathrm{AB}$, et al. TRPV4 initiates the acute calcium-dependent permeability increase during ventilator-induced lung injury in isolated mouse lungs. Am J Physiol Lung Cell Mol Physiol. (2007) 293:L923-32. doi: 10.1152/ajplung.00221.2007

30. Rahaman SO, Grove LM, Paruchuri S, Southern BD, Abraham S, Niese KA, et al. TRPV4 mediates myofibroblast differentiation and pulmonary fibrosis in mice. J Clin Invest. (2014) 124:5225-38. doi: 10.1172/JCI75331

31. Nayak PS, Wang Y, Najrana T, Priolo LM, Rios M, Shaw SK, et al. Mechanotransduction via TRPV4 regulates inflammation and differentiation in fetal mouse distal lung epithelial cells. Respir Res. (2015) 16:60. doi: 10.1186/s12931-015-0224-4

32. Goldenberg NM, Wang L, Ranke H, Liedtke W, Tabuchi A, Kuebler WM. TRPV4 is required for hypoxic pulmonary vasoconstriction. Anesthesiology. (2015) 122:1338-48. doi: 10.1097/ALN.0000000000000647

33. Zhao P, Lieu T, Barlow N, Sostegni S, Haerteis S, Korbmacher C, et al. Neutrophil Elastase Activates Protease-activated Receptor-2 (PAR2) and Transient Receptor Potential Vanilloid 4 (TRPV4) to cause inflammation and pain. J Biol Chem. (2015) 290:13875-87. doi: 10.1074/jbc.M115.642736

34. Kung C. A possible unifying principle for mechanosensation. Nature. (2005) 436:647-54. doi: 10.1038/nature03896

35. Christensen AP, Corey DP. TRP channels in mechanosensation: direct or indirect activation? Nat Rev Neurosci. (2007) 8:510-21. doi: 10.1038/nrn2149

36. Brohawn SG, Su Z, MacKinnon R. Mechanosensitivity is mediated directly by the lipid membrane in TRAAK and TREK1 K+ channels. Proc Natl Acad Sci USA. (2014) 111:3614-9. doi: 10.1073/pnas.1320768111

37. Loukin S, Zhou X, Su Z, Saimi Y, Kung C. Wild-type and brachyolmia-causing mutant TRPV4 channels respond directly to stretch force. J Biol Chem. (2010) 285:27176-81. doi: 10.1074/jbc.M110.143370

38. Pedersen SF, Nilius B. Transient receptor potential channels in mechanosensing and cell volume regulation. Meth Enzymol. (2007) 428:183-207. doi: 10.1016/S0076-6879(07)28010-3

39. White JPM, Cibelli M, Urban L, Nilius B, McGeown JG, Nagy I. TRPV4: molecular conductor of a diverse orchestra. Physiol Rev. (2016) 96:91173. doi: 10.1152/physrev.00016.2015

40. Matthews BD, Thodeti CK, Tytell JD, Mammoto A, Overby DR, Ingber DE. Ultra-rapid activation of TRPV4 ion channels by mechanical forces applied to cell surface betal integrins. Integr Biol. (2010) 2:435-42. doi: $10.1039 / \mathrm{c} 0 \mathrm{ib} 00034 \mathrm{e}$

41. Thodeti CK, Matthews B, Ravi A, Mammoto A, Ghosh K, Bracha $\mathrm{AL}$, et al. TRPV4 channels mediate cyclic strain-induced endothelial cell reorientation through integrin-to-integrin signaling. Circ Res. (2009) 104:1123-30. doi: 10.1161/CIRCRESAHA.108.192930

42. Watanabe H, Vriens J, Prenen J, Droogmans G, Voets T, Nilius B. Anandamide and arachidonic acid use epoxyeicosatrienoic acids to activate TRPV4 channels. Nature. (2003) 424:434-8. doi: 10.1038/nature01807
43. Garcia-Elias A, Mrkonjic S, Pardo-Pastor C, Inada H, Hellmich UA, Rubio-Moscardó F, et al. Phosphatidylinositol-4,5-biphosphatedependent rearrangement of TRPV4 cytosolic tails enables channel activation by physiological stimuli. Proc Natl Acad Sci USA. (2013) 110:9553-8. doi: 10.1073/pnas.1220231110

44. Takahashi N, Hamada-Nakahara S, Itoh Y, Takemura K, Shimada A, Ueda Y, et al. TRPV4 channel activity is modulated by direct interaction of the ankyrin domain to PI(4,5)P2. Nat Commun. (2014) 5:4994. doi: 10.1038/ncomms 5994

45. Berna-Erro A, Izquierdo-Serra M, Sepúlveda RV, Rubio-Moscardo F, DoñateMacián P, Serra SA, et al. Structural determinants of 5',6'-epoxyeicosatrienoic acid binding to and activation of TRPV4 channel. Sci Rep. (2017) 7:10522. doi: 10.1038/s41598-017-11274-1

46. Gao X, Wu L, O'Neil RG. Temperature-modulated diversity of TRPV4 channel gating: activation by physical stresses and phorbol ester derivatives through protein kinase C-dependent and -independent pathways. J Biol Chem. (2003) 278:27129-37. doi: 10.1074/jbc.M302517200

47. Fan H-C, Zhang X, McNaughton PA. Activation of the TRPV4 ion channel is enhanced by phosphorylation. J Biol Chem. (2009) 284:2788491. doi: $10.1074 /$ jbc.M109.028803

48. Peng H, Lewandrowski U, Müller B, Sickmann A, Walz G, Wegierski T. Identification of a Protein Kinase C-dependent phosphorylation site involved in sensitization of TRPV4 channel. Biochem Biophys Res Commun. (2010) 391:1721-5. doi: 10.1016/j.bbrc.2009.12.140

49. Arniges M, Fernández-Fernández JM, Albrecht N, Schaefer M, Valverde MA. Human TRPV4 channel splice variants revealed a key role of ankyrin domains in multimerization and trafficking. J Biol Chem. (2006) 281:15806. doi: 10.1074/jbc.M511456200

50. Lee EJ, Shin SH, Hyun S, Chun J, Kang SS. Mutation of a putative S-nitrosylation site of TRPV4 protein facilitates the channel activates. Animal Cells Syst. (2011) 15:95-106. doi: 10.1080/19768354.2011.555183

51. Xu H, Fu Y, Tian W, Cohen DM. Glycosylation of the osmoresponsive transient receptor potential channel TRPV4 on Asn-651 influences membrane trafficking. Am J Physiol Renal Physiol. (2006) 290:F1103-9. doi: 10.1152/ajprenal.00245.2005

52. Smith JB, Knowlton RP, Agarwal SS. Human lymphocyte responses are enhanced by culture at 40 degrees C. J Immunol. (1978) 121:691-4.

53. Tournier J-N, Hellmann AQ, Lesca G, Jouan A, Drouet E, Mathieu J. Feverlike thermal conditions regulate the activation of maturing dendritic cells. $J$ Leukoc Biol. (2003) 73:493-501. doi: 10.1189/jlb.1002506

54. Tominaga M, Caterina MJ. Thermosensation and pain. J Neurobiol. (2004) 61:3-12. doi: 10.1002/neu.20079

55. Todaka H, Taniguchi J, Satoh J, Mizuno A, Suzuki M. Warm temperaturesensitive transient receptor potential vanilloid 4 (TRPV4) plays an essential role in thermal hyperalgesia. J Biol Chem. (2004) 279:351338. doi: $10.1074 /$ jbc.M406260200

56. Majhi RK, Sahoo SS, Yadav M, Pratheek BM, Chattopadhyay S, Goswami C. Functional expression of TRPV channels in T cells and their implications in immune regulation. FEBS J. (2015) 282:2661-81. doi: 10.1111/febs.13306

57. Mancarella S, Wang Y, Gill DL. Signal transduction: STIM1 senses both $\mathrm{Ca}^{2+}$ and heat. Nat Chem Biol. (2011) 7:344-5. doi: 10.1038/nchembio.587

58. Spinsanti G, Zannolli R, Panti C, Ceccarelli I, Marsili L, Bachiocco V, et al. Quantitative Real-Time PCR detection of TRPV1-4 gene expression in human leukocytes from healthy and hyposensitive subjects. Mol Pain. (2008) 4:51. doi: 10.1186/1744-8069-4-51

59. Balakrishna S, Song W, Achanta S, Doran SF, Liu B, Kaelberer MM, et al. TRPV4 inhibition counteracts edema and inflammation and improves pulmonary function and oxygen saturation in chemically induced acute lung injury. Am J Physiol Lung Cell Mol Physiol. (2014) 307:L15872. doi: 10.1152/ajplung.00065.2014

60. Morty RE, Kuebler WM. TRPV4: an exciting new target to promote alveolocapillary barrier function. Am J Physiol Lung Cell Mol Physiol. (2014) 307:L817-21. doi: 10.1152/ajplung.00254.2014

61. Schmidt EP, Kuebler WM, Lee WL, Downey GP. Adhesion molecules: master controllers of the circulatory system. Compr Physiol. (2016) 6:94573. doi: $10.1002 /$ cphy.c150020

62. Narita K, Sasamoto S, Koizumi S, Okazaki S, Nakamura H, Inoue T, et al. TRPV4 regulates the integrity of the blood-cerebrospinal fluid barrier 
and modulates transepithelial protein transport. FASEB J. (2015) 29:224759. doi: 10.1096/fj.14-261396

63. Villalta PC, Rocic P, Townsley MI. Role of MMP2 and MMP9 in TRPV4induced lung injury. Am J Physiol Lung Cell Mol Physiol. (2014) 307:L6529. doi: 10.1152/ajplung.00113.2014

64. Yin J, Hoffmann J, Kaestle SM, Neye N, Wang L, Baeurle J, et al. Negativefeedback loop attenuates hydrostatic lung edema via a cGMP-dependent regulation of transient receptor potential vanilloid 4. Circ Res. (2008) 102:96674. doi: 10.1161/CIRCRESAHA.107.168724

65. Alvarez DF, King JA, Weber D, Addison E, Liedtke W, Townsley MI. Transient receptor potential vanilloid 4-mediated disruption of the alveolar septal barrier: a novel mechanism of acute lung injury. Circ Res. (2006) 99:988-95. doi: 10.1161/01.RES.0000247065.11756.19

66. Jian M-Y, King JA, Al-Mehdi A-B, Liedtke W, Townsley MI. High vascular pressure-induced lung injury requires P450 epoxygenase-dependent activation of TRPV4. Am J Respir Cell Mol Biol. (2008) 38:38692. doi: $10.1165 / \mathrm{rcmb} .2007-0192 \mathrm{OC}$

67. Willette RN, Bao W, Nerurkar S, Yue T-L, Doe CP, Stankus G, et al. Systemic activation of the transient receptor potential vanilloid subtype 4 channel causes endothelial failure and circulatory collapse: Part 2. J Pharmacol Exp Ther. (2008) 326:443-52. doi: 10.1124/jpet.107.134551

68. Pairet N, Mang S, Fois G, Keck M, Kühnbach M, Gindele J, et al. TRPV4 inhibition attenuates stretch-induced inflammatory cellular responses and lung barrier dysfunction during mechanical ventilation. PLoS ONE. (2018) 13:e0196055. doi: 10.1371/journal.pone.0196055

69. Alpizar YA, Boonen B, Sanchez A, Jung C, López-Requena A, Naert $\mathrm{R}$, et al. TRPV4 activation triggers protective responses to bacterial lipopolysaccharides in airway epithelial cells. Nat Commun. (2017) 8:1059. doi: 10.1038/s41467-017-01201-3

70. Schneider B, Gutbier B, Reppe K, Lienau J, Michalik L, Erfinanda L, et al. Role of transient receptor potential Vanilloid 4 for development of acute lung injury in pneumococcal pneumonia. Pneumologie. (2018) 72:226. doi: 10.1055/s-0037-1615314

71. Chiu IM, Heesters BA, Ghasemlou N, Von Hehn CA, Zhao F, Tran J, et al. Bacteria activate sensory neurons that modulate pain and inflammation. Nature. (2013) 501:52-7. doi: 10.1038/nature12479

72. Wallecha A, Wood L, Pan Z-K, Maciag PC, Shahabi V, Paterson Y. Listeria monocytogenes-derived listeriolysin $\mathrm{O}$ has pathogen-associated molecular pattern-like properties independent of its hemolytic ability. Clin Vaccine Immunol. (2013) 20:77-84. doi: 10.1128/CVI.00488-12

73. Scheraga RG, Abraham S, Grove LM, Southern BD, Crish JF, Perelas A, et al. TRPV4 Protects the lung from bacterial pneumonia via MAPK molecular pathway switching. J Immunol. (2020) 204:1310-21. doi: 10.4049/jimmunol.1901033

74. Rayees S, Joshi JC, Tauseef M, Anwar M, Baweja S, Rochford I, et al. PAR2mediated cAMP generation suppresses TRPV4-dependent $\mathrm{Ca}^{2+}$ signaling in alveolar macrophages to resolve TLR4-induced inflammation. Cell Rep. (2019) 27:793-805.e4. doi: 10.1016/j.celrep.2019.03.053

75. Suzuki T, Yamashita C, Zemans RL, Briones N, Van Linden A, Downey GP. Leukocyte elastase induces lung epithelial apoptosis via a PAR-1-, NFkappaB-, and p53-dependent pathway. Am J Respir Cell Mol Biol. (2009) 41:742-55. doi: 10.1165/rcmb.2008-0157OC

76. Maharshak N, Huh EY, Paiboonrungruang C, Shanahan M, Thurlow L, Herzog J, et al. Enterococcus faecalis gelatinase mediates intestinal permeability via protease-activated receptor 2. Infect Immun. (2015) 83:276270. doi: 10.1128/IAI.00425-15

77. Richmond BW, Brucker RM, Han W, Du R-H, Zhang Y, Cheng D-S, et al. Airway bacteria drive a progressive COPD-like phenotype in mice with polymeric immunoglobulin receptor deficiency. Nat Commun. (2016) 7:11240. doi: $10.1038 /$ ncomms 11240

78. Hou H-H, Wang H-C, Cheng S-L, Chen Y-F, Lu K-Z, Yu C-J. MMP-12 activates protease-activated receptor-1, upregulates placenta growth factor, and leads to pulmonary emphysema. Am J Physiol Lung Cell Mol Physiol. (2018) 315:L432-42. doi: 10.1152/ajplung.00216.2017

79. Walsh SW, Nugent WH, Solotskaya AV, Anderson CD, Grider JR, Strauss JF. Matrix Metalloprotease-1 and elastase are novel uterotonic agents acting through protease-activated receptor 1. Reprod Sci. (2018) 25:105866. doi: $10.1177 / 1933719117732162$
80. Chen H, Qu J, Huang X, Kurundkar A, Zhu L, Yang N, et al. Mechanosensing by the $\alpha 6$-integrin confers an invasive fibroblast phenotype and mediates lung fibrosis. Nat Commun. (2016) 7:12564. doi: 10.1038/ncomms12564

81. Discher DE, Janmey P, Wang Y-L. Tissue cells feel and respond to the stiffness of their substrate. Science. (2005) 310:1139-43. doi: 10.1126/science.1116995

82. Upadhyaya A. Mechanosensing in the immune response. Semin Cell Dev Biol. (2017) 71:137-45. doi: 10.1016/j.semcdb.2017.08.031

83. Hilscher MB, Sehrawat T, Arab JP, Zeng Z, Gao J, Liu M, et al. Mechanical stretch increases expression of CXCL1 in liver sinusoidal endothelial cells to recruit neutrophils, generate sinusoidal microthombi, and promote portal hypertension. Gastroenterology. (2019) 157:193209.e9. doi: 10.1053/j.gastro.2019.03.013

84. Yoshida K, Kondo R, Wang Q, Doerschuk CM. Neutrophil cytoskeletal rearrangements during capillary sequestration in bacterial pneumonia in rats. Am J Respir Crit Care Med. (2006) 174:689-98. doi: 10.1164/rccm.200502-276OC

85. Ekpenyong AE, Toepfner N, Chilvers ER, Guck J. Mechanotransduction in neutrophil activation and deactivation. Biochim Biophys Acta. (2015) 1853:3105-16. doi: 10.1016/j.bbamcr.2015.07.015

86. Bashant KR, Vassallo A, Herold C, Berner R, Menschner L, Subburayalu $\mathrm{J}$, et al. Real-time deformability cytometry reveals sequential contraction and expansion during neutrophil priming. J Leukoc Biol. (2019) 105:114353. doi: 10.1002/JLB.MA0718-295RR

87. Baratchi S, Knoerzer M, Khoshmanesh K, Mitchell A, McIntyre P. Shear stress regulates TRPV4 channel clustering and translocation from adherens junctions to the basal membrane. Sci Rep. (2017) 7:15942. doi: 10.1038/s41598-017-16276-7

88. Wissing TB, van Haaften EE, Koch SE, Ippel BD, Kurniawan NA, Bouten CVC, et al. Hemodynamic loads distinctively impact the secretory profile of biomaterial-activated macrophages - implications for in situ vascular tissue engineering. Biomater Sci. (2019) 8:132-47. doi: 10.1039/C9BM0 $1005 \mathrm{~J}$

89. Tabuchi A, Kuebler WM. Alveolar tidal flooding - a new mechanism of ventilator-induced lung injury? In: D105 Critical Care: Ventilator Induced Lung Injury and ARDS - From Mice to Biomarkers in ARDS. American Thoracic Society International Conference Abstracts. San Diego, CA: American Thoracic Society (2018), A7517.

90. Arciniegas E, Neves CY, Carrillo LM, Zambrano EA, Ramírez R. Endothelial-mesenchymal transition occurs during embryonic pulmonary artery development. Endothelium. (2005) 12:193200. doi: 10.1080/10623320500227283

91. Sicard D, Haak AJ, Choi KM, Craig AR, Fredenburgh LE, Tschumperlin DJ. Aging and anatomical variations in lung tissue stiffness. Am J Physiol Lung Cell Mol Physiol. (2018) 314:L946-55. doi: 10.1152/ajplung.004 15.2017

92. Barriga EH, Franze K, Charras G, Mayor R. Tissue stiffening coordinates morphogenesis by triggering collective cell migration in vivo. Nature. (2018) 554:523-7. doi: 10.1038/nature25742

93. Dieffenbach PB, Maracle $M$, Tschumperlin DJ, Fredenburgh LE. Mechanobiological feedback in pulmonary vascular disease. Front Physiol. (2018) 9:951. doi: 10.3389/fphys.2018.00951

94. Arora PD, Di Gregorio M, He P, McCulloch CA. TRPV4 mediates the $\mathrm{Ca}^{2+}$ influx required for the interaction between flightless-1 and nonmuscle myosin, and collagen remodeling. J Cell Sci. (2017) 130:2196208. doi: $10.1242 /$ jcs. 201665

95. Sharma S, Goswami R, Zhang DX, Rahaman SO. TRPV4 regulates matrix stiffness and TGF $\beta 1$-induced epithelial-mesenchymal transition. J Cell Mol Med. (2019) 23:761-74. doi: 10.1111/jcmm.13972

96. Gilchrist CL, Leddy HA, Kaye L, Case ND, Rothenberg KE, Little $\mathrm{D}$, et al. TRPV4-mediated calcium signaling in mesenchymal stem cells regulates aligned collagen matrix formation and vinculin tension. Proc Natl Acad Sci USA. (2019) 116:1992-7. doi: 10.1073/pnas.18110 95116

97. Henry CO, Dalloneau E, Pérez-Berezo M-T, Plata C, Wu Y, Guillon A, et al. In vitro and in vivo evidence for an inflammatory role of the calcium channel TRPV4 in lung epithelium: potential involvement in cystic fibrosis. Am J Physiol Lung Cell Mol Physiol. (2016) 311:L664-75. doi: 10.1152/ajplung.00442.2015 
98. Sonkusare SK, Bonev AD, Ledoux J, Liedtke W, Kotlikoff MI, Heppner TJ, et al. Elementary $\mathrm{Ca}^{2+}$ signals through endothelial TRPV4 channels regulate vascular function. Science. (2012) 336:597-601. doi: 10.1126/science. 1216283

99. Hong K, Cope EL, DeLalio LJ, Marziano C, Isakson BE, Sonkusare SK. TRPV4 (transient receptor potential vanilloid 4) channel-dependent negative feedback mechanism regulates $\mathrm{Gq}$ protein-coupled receptor-induced vasoconstriction. Arterioscler Thromb Vasc Biol. (2018) 38:542-54. doi: 10.1161/ATVBAHA.117. 310038
Conflict of Interest: The authors declare that the research was conducted in the absence of any commercial or financial relationships that could be construed as a potential conflict of interest.

Copyright () 2020 Michalick and Kuebler. This is an open-access article distributed under the terms of the Creative Commons Attribution License (CC BY). The use, distribution or reproduction in other forums is permitted, provided the original author(s) and the copyright owner(s) are credited and that the original publication in this journal is cited, in accordance with accepted academic practice. No use, distribution or reproduction is permitted which does not comply with these terms. 